\title{
Metabolomic Characterization of Laborers Exposed to Welding Fumes
}

\author{
Ching-Hua Kuo, ${ }^{\dagger, \ddagger, \bigcirc}$ Kuo-Ching Wang, ${ }^{\ddagger}, \S, \bigcirc$ Tze-Feng Tian, ${ }^{\ddagger}, \|$ Mong-Hsun Tsai, ${ }^{\perp}$ Yin-Mei Chiung, ${ }^{\#}$ \\ Chun-Ming Hsiech, ${ }^{\#}$ Sung-Jeng Tsai, ${ }^{\dagger},+\frac{+}{+}$ San-Yuan Wang, ${ }^{\ddagger}, \|$ Dong-Ming Tsai, ${ }^{\ddagger, \|}$ Chiang-Ching Huang, ${ }^{\nabla}$ \\ and Y. Jane Tseng ${ }^{*}, \dagger, \$, \|^{\circ}$
}

${ }^{\dagger}$ Department of Pharmacy, ${ }^{\ddagger}$ Metabolomics Group, ${ }^{\S}$ Graduate Institute of Biomedical Electronics and Bioinformatics, "Department of Computer Science and Information Engineering, and ${ }^{\perp}$ Institute of Biotechnology, National Taiwan University, No. 1, Sec. 4, Roosevelt Road, Taipei, 106, Taiwan

\#Institute of Occupational Safety and Health, Council of Labor Affairs, No. 99, Lane 407, Hengke Road, New Taipei City, Taiwan

${ }^{\nabla}$ Department of Preventive Medicine, Northwestern University, Chicago, Illinois 60611-4402, United States

\section{Supporting Information}

ABSTRACT: The complex composition of welding fumes, multiplicity of molecular targets, diverse cellular effects, and lifestyles associated with laborers vastly complicate the assessment of welding fume exposure. The urinary metabolomic profiles of 35 male welders and 16 male office workers at a Taiwanese shipyard were characterized via ${ }^{1} \mathrm{H}$ NMR spectroscopy and pattern recognition methods. Blood samples for the same 51 individuals were also collected, and the expression levels of the cytokines and other inflammatory markers were examined. This study dichotomized the welding exposure variable into high (welders) versus low (office workers) exposures to examine the differences of continuous outcome markers-metabolites and inflammatory markersbetween the two groups. Fume particle assessments showed that welders were exposed to different concentrations of chromium, nickel, and manganese particles. Multivariate statistical analysis of urinary metabolomic patterns showed higher levels of glycine, taurine, betaine/TMAO, serine, $S$-sulfocysteine, hippurate, gluconate, creatinine, and

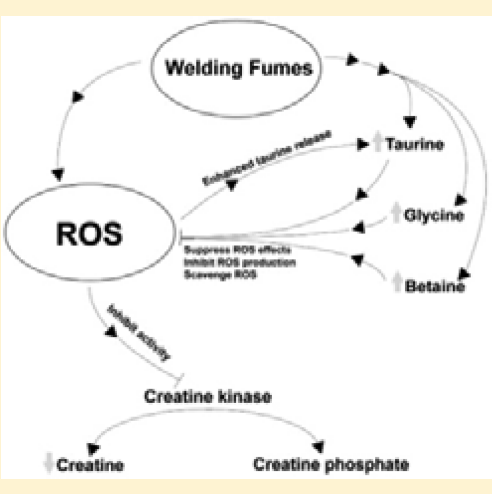
acetone and lower levels of creatine among welders, while only TNF- $\alpha$ was significantly associated with welding fume exposure among all cytokines and other inflammatory markers measured. Of the identified metabolites, the higher levels of glycine, taurine, and betaine among welders were suspected to play some roles in modulating inflammatory and oxidative tissue injury processes. In this metabolomics experiment, we also discovered that the association of the identified metabolites with welding exposure was confounded by smoking, but not with drinking, which is a finding consistent with known modified response of inflammatory markers among smokers. Our results correspond with prior studies that utilized nonmetabolomic analytical techniques and suggest that the metabolomic profiling is an efficient method to characterize the overall effect of welding fume exposure and other confounders.

\section{INTRODUCTION}

Metabolomics with nuclear magnetic resonance (NMR) or gas/ liquid chromatography-mass spectrometry (GC/LC-MS) can detect and quantitatively measure the endogenous and exogenous low molecular weight metabolites in the investigation of metabolic disturbances in cells, tissues, and organisms. Patterns of these large quantities of metabolites are able to provide considerable insight into disregulated metabolic pathways, underlying disease processes, and pathophysiological changes induced by external stimuli such as environmental stress, drugs, nutrition, or genetic modification. ${ }^{1}$ Because of the analytical advantages of metabolomic analysis, it is unsurprising that metabolomics has already been widely applied in the field of toxicology, with specific regards to studying hepatotoxicity, neurotoxicity, renal toxicity, and pulmonary toxicity. ${ }^{2-5}$ Moreover, the ability of metabolomic analysis to characterize and capture the overall influence of net chemical mixtures (including interactive effects) and spatial/ temporal heterogeneity in contaminant distribution on living organisms draws attention to its future value and use in the emerging field of environmental risk assessment. ${ }^{6,7}$

The effect of air pollutants produced by welding processes on the health of laborers, specifically welders, is a major concern of occupational medicine..$^{8-10}$ In addition to work-related injuries, welders often suffer from acute and chronic occupational diseases such as hepatitis, dermatitis, neuropathy, and encephalopathy and are often associated with respiratory conditions such as chronic obstructive pulmonary disease, asthma, chronic bronchitis, and pneumonia after long-term exposure. ${ }^{11-16}$ Welders are also associated with an increased

Received: October 27, 2011

Published: January 31, 2012 
Table 1. Distribution of Participants by Fume Exposure Status and Alcohol and Cigarette Use ${ }^{a}$

\begin{tabular}{|c|c|c|c|c|c|c|c|}
\hline \multicolumn{4}{|c|}{ welders } & \multicolumn{4}{|c|}{ controls } \\
\hline \multirow{2}{*}{\multicolumn{2}{|c|}{$\begin{array}{l}\text { smokers } \\
\text { group C }\end{array}$}} & \multicolumn{2}{|c|}{ nonsmokers } & \multirow{2}{*}{\multicolumn{2}{|c|}{$\begin{array}{l}\text { smokers } \\
\text { group C }\end{array}$}} & \multicolumn{2}{|c|}{ nonsmokers } \\
\hline & & \multirow{2}{*}{$\begin{array}{c}\text { group A } \\
\text { nondrinkers }\end{array}$} & \multirow{2}{*}{$\begin{array}{l}\text { group B } \\
\text { drinkers }\end{array}$} & & & \multirow{2}{*}{$\frac{\text { group A }}{\text { nondrinkers }}$} & \multirow{2}{*}{$\frac{\text { group B }}{\text { drinkers }}$} \\
\hline nondrinkers & drinkers & & & nondrinkers & drinkers & & \\
\hline 5 & 18 & 10 & 2 & 3 & 1 & 6 & 6 \\
\hline \multirow{2}{*}{\multicolumn{2}{|c|}{23}} & \multicolumn{2}{|c|}{12} & & & \multicolumn{2}{|c|}{12} \\
\hline & & \multicolumn{2}{|c|}{35} & \multicolumn{4}{|c|}{16} \\
\hline
\end{tabular}

risk for lung cancer, laryngeal cancer, esophageal cancer, and, potentially, leukemia. ${ }^{17-20}$

Fumes generated by welding processes contain considerable amounts and various types of gases and particulate dust. Gaseous components of welding fumes commonly contain carbon monoxide, nitrogen oxides, and ozone. Depending on the welding technique utilized, particulate components of welding fumes may also contain metal particulates such as manganese, chromium, nickel, vanadium, and their oxides. ${ }^{21-23}$ Heavy metal byproducts are also largely present in welding fumes and are potentially harmful when aspirated. Ultrafine particles have unique toxicological characteristics due to their small size. Welding-produced nanoparticulates are able to translocate from the respiratory tract and ultimately become deposited in other extrapulmonary organs to produce their toxic effect. $^{24}$ These ultrafine particulates and their soluble fractions, as a result, are often associated with occupational diseases observed in welders. ${ }^{25-27}$

Complex toxicants in welding fumes have a wide range of molecular targets and diverse cellular effects. Heavy metals, such as hexavalent chromium, manganese, and nickel, attack lung epithelial cells and activate mitogen-activated protein kinase. $^{28,29}$ Gene expression profiling using suppressionsubtractive hybridization and cDNA microarray in rat mononuclear cells in response to welding fume exposure shows that genes associated with the immune response, transcription factors, and tyrosine kinases were altered. ${ }^{30}$ Welding fumes have also been demonstrated to increase lipid peroxidation in plasma, lung, and liver tissue in rats. ${ }^{31}$ Analysis of genetic polymorphisms and centromeres in micronuclei showed significantly higher levels of chromosome/genome damage in welders. ${ }^{32}$ In one study, DNA damage was associated with higher chromium, lead, and nickel blood and urine concentrations. ${ }^{33}$ In addition, ozone, a strong oxidant produced during welding, generates reactive oxygen species (ROS) and causes oxidative injury in culture cells. ${ }^{34,35}$ Via the oxidative stress mechanism, welding fumes enhance the inflammatory response ${ }^{36}$ and induce cytotoxic reactions such as DNA breakdown and cytokine activation that ultimately result in cellular apoptosis and neuronal degeneration. ${ }^{21,31,37}$

Because of extensive study population heterogeneity and dissimilar site-to-site welding environments, results of existing toxicological studies of human exposure to welding fumes are inherently difficult to interpret. ${ }^{27}$ The toxicity of welding fumes may be a result of the composite interaction of the biological actions of multiple fume components; therefore, findings regarding the biological mechanisms of action of single components of welding fumes may be inconsistent, incomplete, or contradictory from study to study if only investigated at the epidemiological level. Analyses of disease-toxicant interactions may also be further confounded by certain lifestyle behaviors such as smoking. ${ }^{38}$

This study aims to investigate the long-term effect of welding fume exposure on metabolomic profiles. We examined the complex effects of welding fumes via ${ }^{1} \mathrm{H}$ NMR spectroscopy analysis of metabolomic profiles of individuals employed by the shipbuilding company, CSBC Corporation, Taiwan. Fume composition, particle size, and individual immune response were also evaluated. A spot urine sample for ${ }^{1} \mathrm{H}$ NMR analysis and detailed questionnaires evaluating the medical history and existing lifestyle habits such as smoking or alcohol use were obtained from 51 individuals, of which 35 were welders with prior exposure to welding fumes and 16 were office workers with no prior exposure to welding fumes. A multivariate statistical procedure was used to identify metabolites associated with various environmental stimuli such as welding fume exposure, smoking, and alcohol use. Blood samples for the same 51 individuals were also collected, and the expression levels of the cytokines and other inflammatory markers were examined.

\section{EXPERIMENTAL PROCEDURES}

Study Design and Participants' Profiles. This study was approved by the Institutional Review Board of the Institute of Occupational Safety and Health, Council of Labor Affairs, Taiwan. Written informed consent was obtained from each study participant. A total of 51 full time employees at a shipyard (CSBC Corporation, Keelung, Taiwan) consisting of 35 male welders and 16 male office workers (as controls) aging from 45 to 64 were included in this study.

Forty-one out of 51 participants had employment tenures at the shipyard exceeding 20 years. The average length of employment for the remaining 10 participants was 9 years. The 35 participants exposed to welding fumes were all active welders at the shipyard, whereas the 16 controls were located in their respective administration offices away from the welding sites. A self-administered questionnaire was used to collect information on medical history, cardiopulmonary history, smoking history, drinking history (including herbal alcohol consumption), and occupational history. Individuals with a history of respiratory disease and chronic inflammation diseases were preexcluded from this study. Blood and urine samples of all 51 participants were collected for inflammatory biomarker measurement and ${ }^{1} \mathrm{H}$ NMR metabolomic analysis, respectively.

Participants were stratified into four groups: (1) group A (10 welders and 6 controls) contained participants without any use of alcohol and cigarettes, (2) group B (2 welders and 6 controls) contained participants who were alcohol users but not smokers, (3) group C ( 23 welders and 4 controls) contained participants who were smokers (including alcohol drinkers and nondrinkers), and (4) group D (35 welders and 16 controls) contained all participants. See Table 1 for detail numbers in each group.

Exposure Assessment. Air samples were collected at the manufacture and assembly areas of the shipyard for assessing fine particle exposure of welders. Welding fume exposure originated from either shielded metal arc welding, manual metal arc welding with 
Scheme 1. Scheme for ${ }^{1} \mathrm{H}$ NMR Spectral Analysis of Welding Fume Exposure
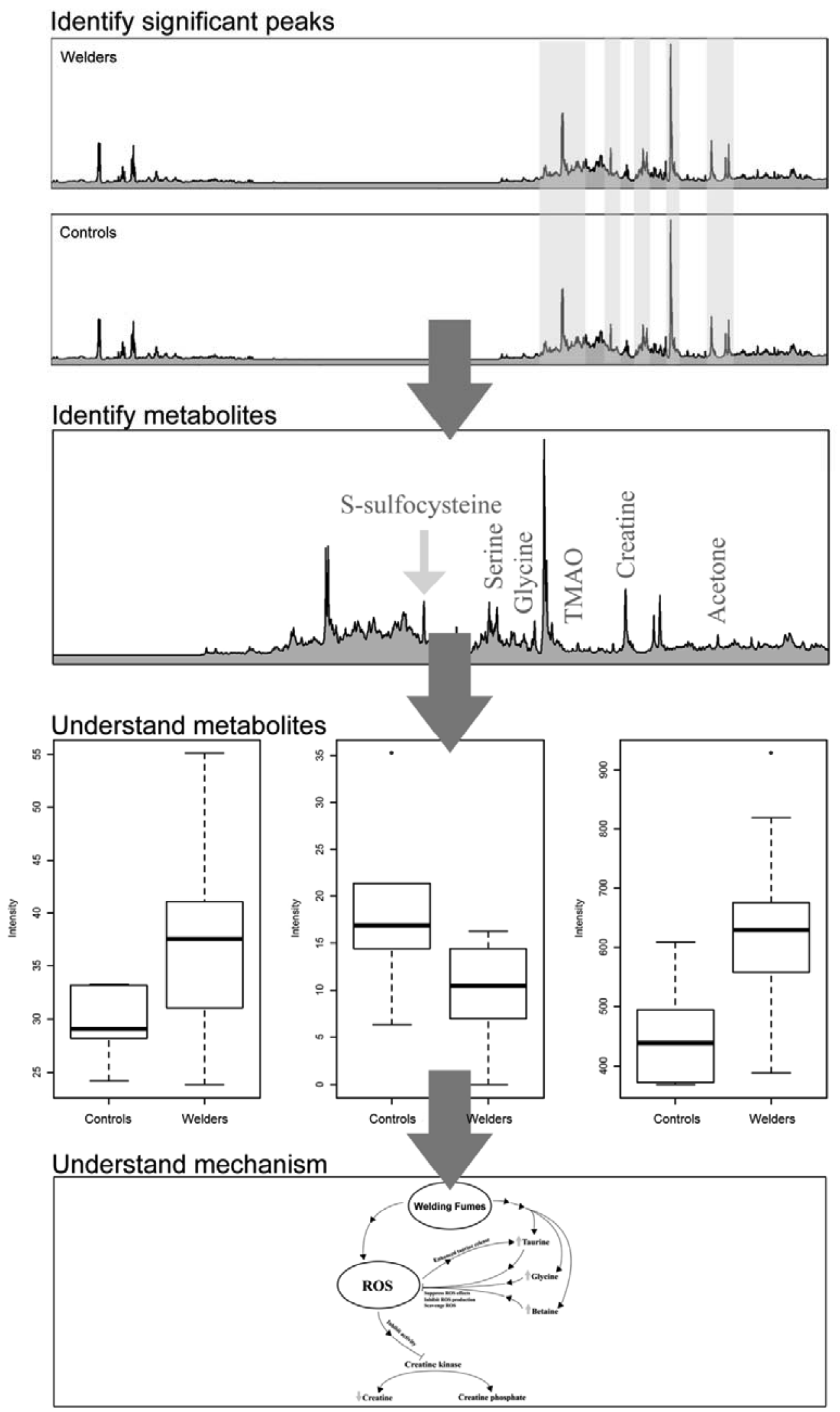

E7016 (0.08\% C, $0.52 \%$ of $\mathrm{Si}, 0.013 \%$ of $\mathrm{P}, 0.004 \%$ of $\mathrm{S}$, and $1.10 \%$ of $\mathrm{Mn})$, or metal inert gas with E71T-1 (0.037\% C, $0.42 \%$ of $\mathrm{Si}, 0.02 \%$ of $\mathrm{P}, 0.010 \%$ of $\mathrm{S}$, and $1.30 \%$ of $\mathrm{Mn}$ ). Fine particulate matter exposure was assessed with "fixed point" and "personal sampling techniques" by
IOSH new cyclone (IOSH, Taipei, Taiwan) and a Nanoparticle Surface Area Monitor (NSAM) (model 3350, TSI, United States) for lung deposited surface area concentration. Air samples were collected on a $37 \mathrm{~mm}$ polyvinylchloride (PVC) or a mixed cellulose ester 
Table 2. Welding Fume Component Concentrations Measured by Different Sampling Types in Shipyard Working Environment

\begin{tabular}{|c|c|c|c|c|c|}
\hline \multicolumn{2}{|c|}{ collection area } & \multicolumn{2}{|c|}{ manufacture } & \multicolumn{2}{|c|}{ assembly } \\
\hline & le counts & \multicolumn{2}{|c|}{14} & \multicolumn{2}{|c|}{6} \\
\hline \multicolumn{2}{|c|}{ sampling type } & fixed point collection & personal sampler collection & fixed point collection & personal sampler collection \\
\hline $\mathrm{Cr}\left(\mathrm{mg} / \mathrm{m}^{3}\right)$ & mean $\pm \mathrm{SD}($ range $)$ & $0.06 \pm 0.02(0.03-0.11)$ & $0.64 \pm 0.35(0.27-1.26)$ & $0.76 \pm 0.97(0.08-2.59)$ & $2.19 \pm 0.88(1.19-3.43)$ \\
\hline $\mathrm{Ni}\left(\mathrm{mg} / \mathrm{m}^{3}\right)$ & mean $\pm \mathrm{SD}$ (range) & $0.02 \pm 0.02(0.01-0.04)$ & $0.18 \pm 0.23(0.01-0.96)$ & $0.07 \pm 0.04(0.01-0.14)$ & $0.41 \pm 0.30(0.08-0.91)$ \\
\hline $\operatorname{Mn}\left(\mathrm{mg} / \mathrm{m}^{3}\right)$ & mean $\pm \mathrm{SD}$ (range) & $0.11 \pm 0.11(0.01-0.26)$ & $0.07 \pm 0.05(0.02-0.19)$ & $1.05 \pm 0.94(0.22-2.29)$ & $1.23 \pm 0.77(0.17-2.26)$ \\
\hline
\end{tabular}

(MEC) membrane filter (SKC, United States) and weighed by a 5decimal microbalance (AX-205, Mettler Toledo, Switzerland). The mass concentration was determined as described in a previous publication. ${ }^{39}$

Inductively coupled plasma mass spectrometry (ICP/MS, 7500CE, Agilent, United States) was utilized to detect chromium, nickel, and manganese concentrations. Babington Nebulizer (PNG1820-60453, Agilent) was used to nebulize the analytes, with $0.3 \mathrm{~mm}$ diameter pump component, and a sample introduction speed of $2 \mathrm{~mL} / \mathrm{min}$ in 0.45 rps. The spray chamber was cooled to $2{ }^{\circ} \mathrm{C}$ to prevent larger particles from entering the plasma torch.

Inflammatory Marker Measurement. Blood samples and nasal lavage fluids were collected from individuals after overnight fasting before work. Nasal lavage fluids were collected with the procedures described by Naclerio et al. ${ }^{40,41}$ Nasal lavage fluids were analyzed for granulocyte, monocyte, and lymphocyte differential counts. Real-time polymerase chain reaction (real-time PCR) was performed to detect expression of cytokines including IL-6, IL-8, TNF- $\alpha$, RRM2, NCF1, and PTGS1 on blood samples. Enzyme-linked immunosorbent assay (ELISA) for IL-6, IL-8, Endo-1, and TNF- $\alpha$ detection was performed by using purified $\mathrm{mAb}$-coated plates. All procedures followed standard protocols provided by R\&D Systems (Minneapolis, MN). Cytokine concentrations were measured using a MRX microplate reader (Dynex Technologies, Chantilly, VA) at $450 \mathrm{~nm}$ (reference, $540 \mathrm{~nm}$ ). Quantitative reverse transcription polymerase chain reaction (RTPCR) was performed using $1 \mu \mathrm{g}$ of total RNA that was reverse transcribed using Superscript II enzyme (Invitrogen, Carlsbad, CA). Following reverse transcription, cDNA samples were used for SYBR Green real-time PCR assay. Real-time PCR was performed using ABI 7300 Real Time PCR System (Foster City, CA) with reagents purchased from Applied Biosystems (Foster City, CA). Primer sequences were designed using Primer Express Software (Applied Biosystems). All data were analyzed using the 7300 Real-Time PCR System (Foster City, CA).

Metabolomics Analysis with ${ }^{1} \mathrm{H}$ NMR Spectroscopy in Urine. The overall analysis procedure is shown in Scheme $1 .{ }^{1} \mathrm{H}$ NMR metabolomic profiles of urine samples were analyzed by the following procedures.

All subjects were studied after overnight fasting. The urine samples were collected in the same manner and time for the exposed and nonexposed subjects. Ten milliliters of a spot urine sample was collected from each individual in the morning before work. After sample collection, sodium azide was added to the urine samples to reach a final concentration of $10 \mathrm{mM}$. Urine samples were then centrifuged at $3000 \mathrm{rpm}$ for $15 \mathrm{~min}$. The supernatant was transferred to an Eppendrof and stored at $-80{ }^{\circ} \mathrm{C}$ before NMR analysis. A test sample of $825 \mu \mathrm{L}$ for each study participant was prepared using $500 \mu \mathrm{L}$ of the urine sample, $250 \mu \mathrm{L}$ of $0.2 \mathrm{M} \mathrm{Na}_{2} \mathrm{HPO}_{4}$ ( $\mathrm{pH} 7.4$ ), and $75 \mu \mathrm{L}$ of sodium 3-trimethylsilyl-1-(2,2,3,3- $\left.d_{4}\right)$ propionate (TSP) in $\mathrm{D}_{2} \mathrm{O}$ (final concentration, $0.1 \mathrm{mg} / \mathrm{mL}$ ). $\mathrm{D}_{2} \mathrm{O}$ provided an NMR lock signal for the NMR spectrometer.

Conventional ${ }^{1} \mathrm{H}$ NMR spectra of the urine samples were obtained from a Bruker Avance 600 spectrometer (Bruker Biospin, Germany) operated at $600.04 \mathrm{MHz}$ at $25^{\circ} \mathrm{C}$. One-dimensional ${ }^{1} \mathrm{H}$ NMR spectra were acquired using a standard NOESYPR1D pulse sequence (recycle delay- $90^{\circ}-t_{1}-90^{\circ}-t_{\mathrm{m}}-90^{\circ}$-acquisition; XWIN-NMR3.5) with a recycle delay time of $2 \mathrm{~s}$ and a mixing time of $150 \mathrm{~ms}$. The $90^{\circ}$ pulse length was adjusted to $\sim 12.5 \mu \mathrm{s}$ at $-1 \mathrm{~dB}$, and $t_{1}$ was set to $3 \mu \mathrm{s}$, which provided an acquisition time of $2.72 \mathrm{~s}$. The FIDs were multiplied by an exponential weighting function corresponding to a line broadening of
$0.3 \mathrm{~Hz}$, and the data were zero-filled to $64 \mathrm{k}$ data points. All spectra were corrected for phase and baseline distortions and referenced to the internal reference standard TSP $\left(\delta^{1} \mathrm{H}=0.0\right)$. All spectra were exported and processed by using $\mathrm{R}$ statistical package (version 2.9.1). Each ${ }^{1} \mathrm{H}$ NMR spectrum was segmented and binned with a binning size 0.04 $\mathrm{ppm}$ in the range of chemical shift $\delta 0-10 \mathrm{ppm}$. The integral value of each binned region was calculated, and the integral values contributed to an intensity distribution of the whole spectrum. The peak region of water signal between $\delta 4.5$ and $6.0 \mathrm{ppm}$ was removed, and a total of 212 bins of each spectrum were used for statistical analysis.

Identification of Metabolites and Statistical Analysis. The urine metabolomic profiles of group A (participants without alcohol and cigarette consumption) were initially investigated to identify metabolite biomarkers of welding fume exposure and to avoid the potential confounding factors from alcohol and cigarette use.

Before metabolite identification, all binned NMR spectral data were logged and normalized with a quantile normalization procedure using the Bioconductor package: affy. Identification of differential chemical signals (i.e., binned variables) was performed using two sample $t$ test between the welders and the controls. To assess the probability of false positive findings, we used the positive False Discovery Rate (pFDR) method proposed by Storey. ${ }^{42}$ The pFDR method was applied to calculate $q$ values, the pFDR analogue of the $p$ value, for each test statistic using the Bioconductor package: QVALUE. Binned NMR spectral data with $p$ values less than 0.05 and FDR less than $20 \%$ were considered significant. Significant metabolites were identified by assigning significant NMR spectral data with respect to the internal reference NMR spectra and using the software, Chenomx (Version 7.0, Chenomx Inc., Canada). The metabolites were confirmed by the 2D NMR method, ${ }^{1} \mathrm{H}-{ }^{1} \mathrm{H}$ homonuclear correlation spectroscopy (COSY).

Principal component analysis (PCA) ${ }^{43}$ was performed using the significant variables. PCA is a data reduction technique that aims to identify patterns and similarity among data samples. PCA uses a linear transformation to convert data into new variables called principal components (PC) with each PC orthogonal and uncorrelated to each other. The first PC captures the most variability in the data, and each successive PC has the highest variance unexplained by the preceding PCs. The identified metabolites were also investigated among the welders and controls in group B, group C, and all 51 participants to understand the impacts of alcohol and cigarette use.

\section{RESULTS}

Particle Exposure Assessment. The total welding fume exposure of welders ranged from a minimum value of 0.38 to a maximum value of $2.40 \mathrm{mg} / \mathrm{m}^{3}$ (permissible exposure limit in Taiwan is $10 \mathrm{mg} / \mathrm{m}^{3}$ ), which was significantly greater than that of the controls $\left(0.05 \mathrm{mg} / \mathrm{m}^{3}\right)$. Table 2 showed the exposure concentrations of chromium, nickel, and manganese particles sampled with fixed point and personal sampling techniques at two different shipyard areas. The particle concentration of chromium, mainly produced from welding the stainless steel materials, was the highest among three types of particles. The particle concentrations of manganese are mainly produced from the welding electrodes E7016 and E71T-1. Fixed point sampling concentration measurements of chromium and nickel ranged from 0.03 to 0.11 and from 0.01 to $0.04 \mathrm{mg} / \mathrm{m}^{3}$, 
Table 3. Urinary Metabolites That Show Significantly Different Concentration between Welders and Controls without the Use of Alcohol or Cigarettes (Group A) ${ }^{a}$

\begin{tabular}{|c|c|c|c|c|}
\hline \multirow[b]{2}{*}{ metabolite } & \multicolumn{2}{|c|}{ mean $\pm S D$} & \multirow[b]{2}{*}{ decreasing } & \multirow[b]{2}{*}{ increasing } \\
\hline & intensities among welders & intensities among controls & & \\
\hline creatine $(\mathrm{V})$ & $9.9 \pm 5.4$ & $18.5 \pm 9.6$ & ○०० & ○૦० \\
\hline taurine & $21.0 \pm 6.1$ & $12.9 \pm 4.3$ & ○०० & 000 \\
\hline trimethylamine- $\mathrm{N}$-oxide/betaine & $102.4 \pm 30.5$ & $70.3 \pm 20.4$ & ○০০ & $\bullet \circ 0$ \\
\hline acetone & $38.1 \pm 9.5$ & $29.5 \pm 3.4$ & ○০০ & $\bullet \circ 0$ \\
\hline creatinine & $637.4 \pm 151.9$ & $453.5 \pm 89.4$ & 000 & 000 \\
\hline glycine & $39.1 \pm 8.3$ & $30.1 \pm 2.1$ & ○০০ & $\bullet \circ 0$ \\
\hline gluconate & $91.6 \pm 22.4$ & $62.2 \pm 7.7$ & ○০০ & $\bullet \circ 0$ \\
\hline hippurate & $93.7 \pm 14.3$ & $69.6 \pm 11.3$ & ○০০ & $\bullet \circ 0$ \\
\hline$S$-sulfocystein & $37.8 \pm 8.1$ & $21.1 \pm 7.6$ & ○०० & 000 \\
\hline serine & $135.5 \pm 24.2$ & $84.3 \pm 9.1$ & 000 & $\bullet \circ$ \\
\hline
\end{tabular}

${ }^{a}$ Note the $p$ value of metabolite (welders vs controls). The first three circles indicate decreased concentration in welders, and the last three circles indicate increased concentration in welders. $0, p$ value $<0.05$; 0, $p$ value $<0.01$; and 0 ○, $p$ value $<0.001$.

PC 1

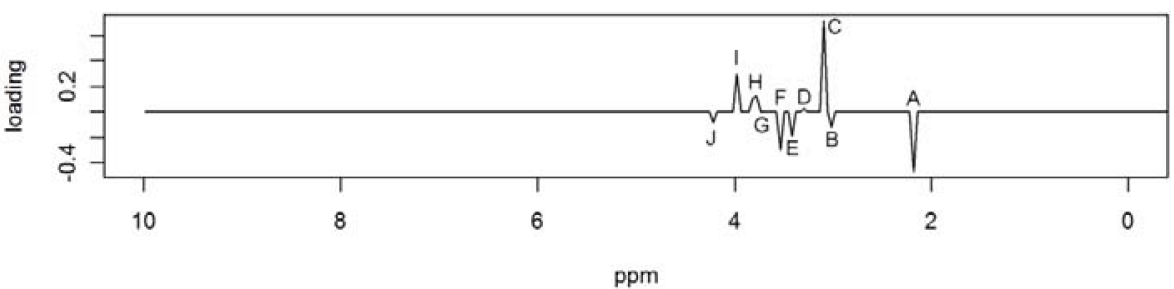

PC 2

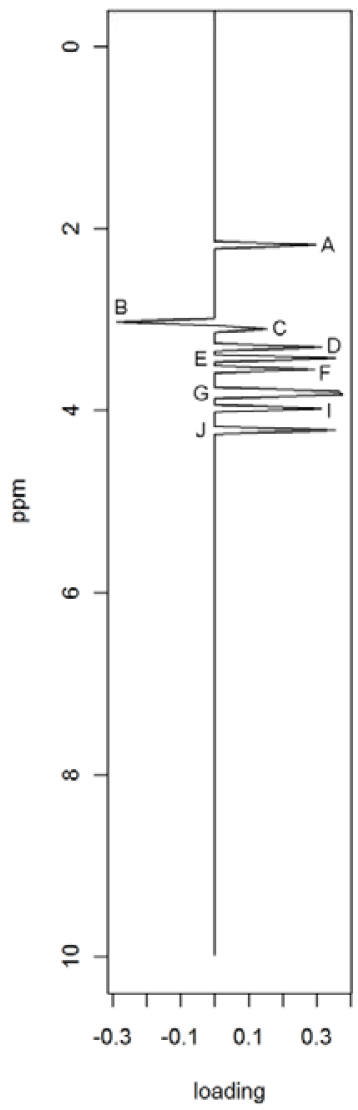

Principal Component Analysis

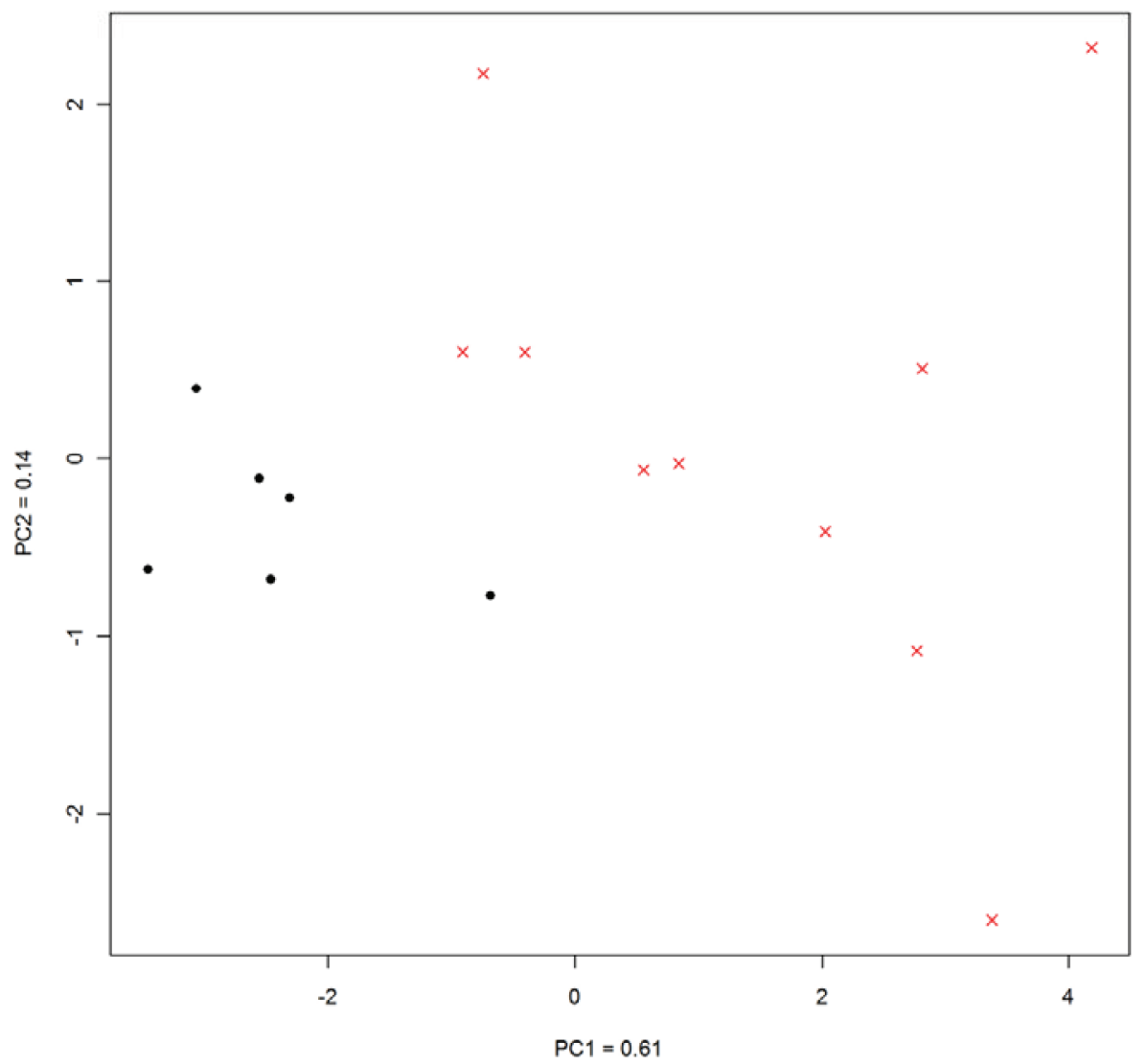

Figure 1. PCA and score plot of significant NMR peaks of group A, 16 participants' urine samples. $\bullet$, controls; $\times$, welders; A, acetone; B, creatine; C, creatinine; D, TMAO/betaine; E, taurine; F, glycine; G, gluconate; H, serine; I, hippurate; and J, S-sulfocysteine. 

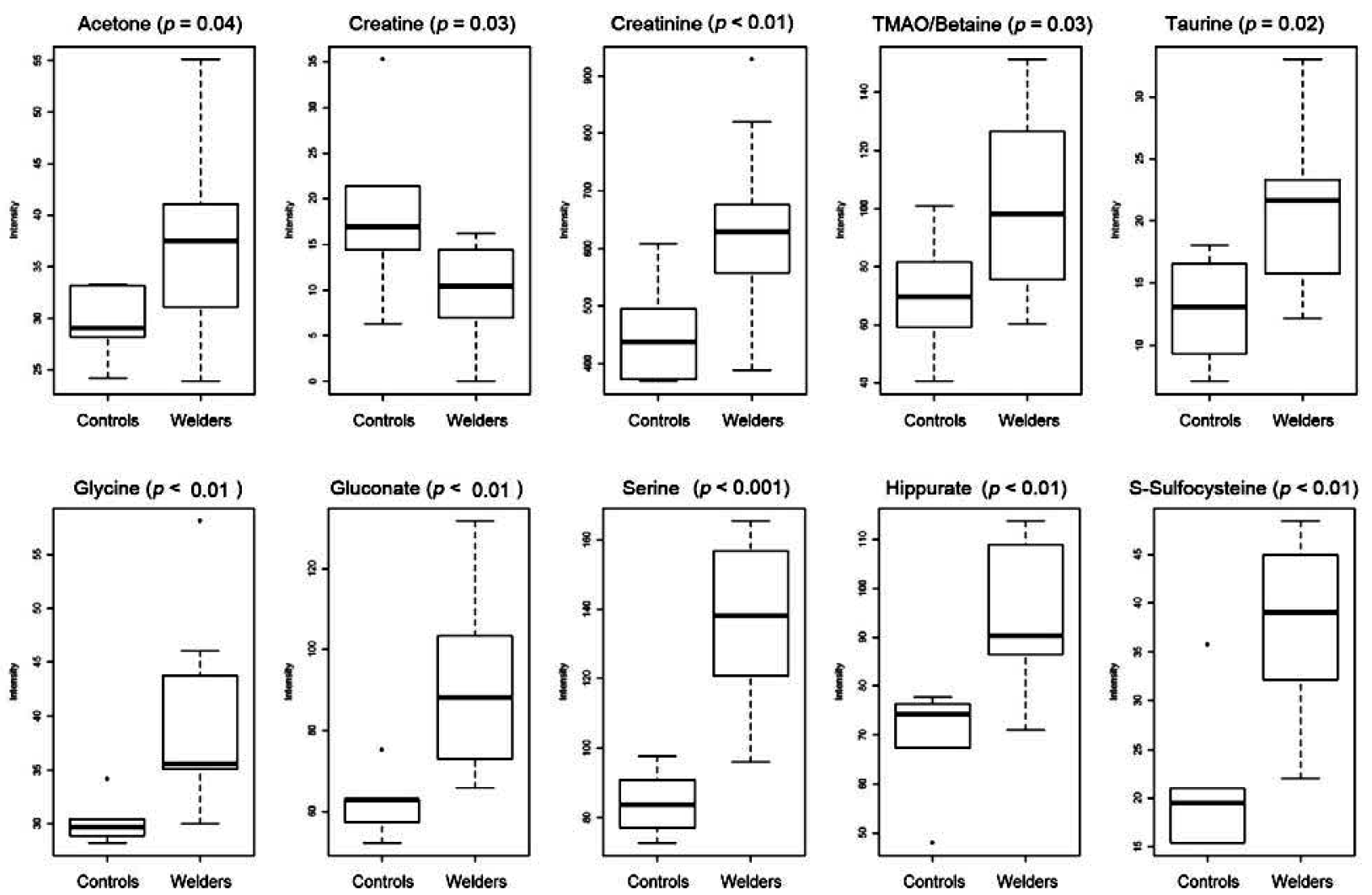

Figure 2. Box plots of intergrated signal intensities of 10 significant metabolites in welders and controls refraining from alcohol consumption and smoking (group A, 10 welders and 6 controls).

respectively, which were less than the fixed point sampling average concentration of manganese at the manufacture area $(p$ $=0.13$ for chromium vs manganese; $p<0.01$ for nickel vs manganese, $t$ test). A high concentration of chromium particles, ranging from 1.19 to $3.43 \mathrm{mg} / \mathrm{m}^{3}$, was measured at the assembly area with personal sampler collection. Exposure measurements for chromium and nickel measured via personal particle sampler were greater than their respective fixed point sampling measurements $(p<0.001$ and $p=0.02$ for chromium at manufacture and assembly areas, respectively; $p=0.02$ and $p$ $=0.04$ for nickel at manufacture and assembly areas, respectively, $t$ test). Concentrations of chromium, nickel, and manganese particles are all higher at the assembly area than at the manufacture area $(p=0.14$ and $p<0.01$ for chromium via fixed point and personal samplers, respectively; $p=0.03$ and $p$ $=0.08$ for nickel via fixed point and personal samplers, respectively; $p=0.06$ and $p=0.01$ for manganese via fixed point and personal samplers, respectively, $t$ test). The concentration of surface area deposition of welding fumes detected by TSI nanoparticle surface area monitor was 4463 $\mu \mathrm{m}^{2} / \mathrm{cm}^{3}$.

Difference of Inflammatory Markers among Welders and Controls. In the nasal lavage analysis, leukocytes, granulocytes, monocytes, and lymphocytes were detectable in most participants. There were no significant changes in these cell counts in the nasal lavage fluids between the welders and the controls in group A. Among all cytokine assays including ELISA for IL-6, IL-8, Endo-1, and TNF- $\alpha$ and RT-PCR on IL6, IL-8, TNF- $\alpha$, Rrm2, NCF1, and PTGS1, only TNF- $\alpha$ displayed distinct expression levels between the welders and the controls in group A ( $p=0.007, t$ test $)$.
NMR Spectral Analysis of Exposure to Welding Fumes. Identification of differential chemical signals between the welders and the controls was performed using two-sample $t$ test. After differential chemical signals were identified, metabolites were assigned according to those chemical signals. To assess the probability of false positive findings, we used the positive False Discovery Rate (pFDR) method proposed by Storey. ${ }^{42}$ Table 3 shows the nominal $p$ value of 10 significant $(p$ $<0.05$ and $\mathrm{FDR}<20 \%)$ metabolites associated with welding fume exposure in group A (participants without any use of alcohol and cigarettes). These metabolites include hippurate (3.97, 7.83, 7.64, and $7.55 \mathrm{ppm}), S$-sulfocysteine (4.24 ppm), creatinine (3.07 and $4.12 \mathrm{ppm})$, serine $(3.82 \mathrm{ppm})$, gluconate (3.77 ppm), glycine (3.57 ppm), taurine (3.44 ppm), TMAO/ betaine $(3.27 \mathrm{ppm})$, creatine $(3.04 \mathrm{ppm})$, and acetone $(2.20$ $\mathrm{ppm})$. Using the signal intensities of these 10 metabolites, PCA of the NMR spectra in group A showed a good separation between the welders and the controls (Figure 1). The distribution of the welding samples tended to be more dispersed when compared with the control samples, suggesting a varying impact of welding fumes on the metabolic responses among the welders. Principal component 1 (PC1) accounted for a high proportion $(60.61 \%)$ of the total variance, while principal component 2 (PC2) was attributable to only $13.92 \%$ of the total variability.

The intergrated signal intensities from NMR spectra indicated that most of these 10 significant metabolites were higher in welders than in controls of group A (Figure 2). The creatine was the only metabolite that exhibited a lower concentration in the welders. The difference in serine concentration was the most significant between the welders and the controls (of group A). 

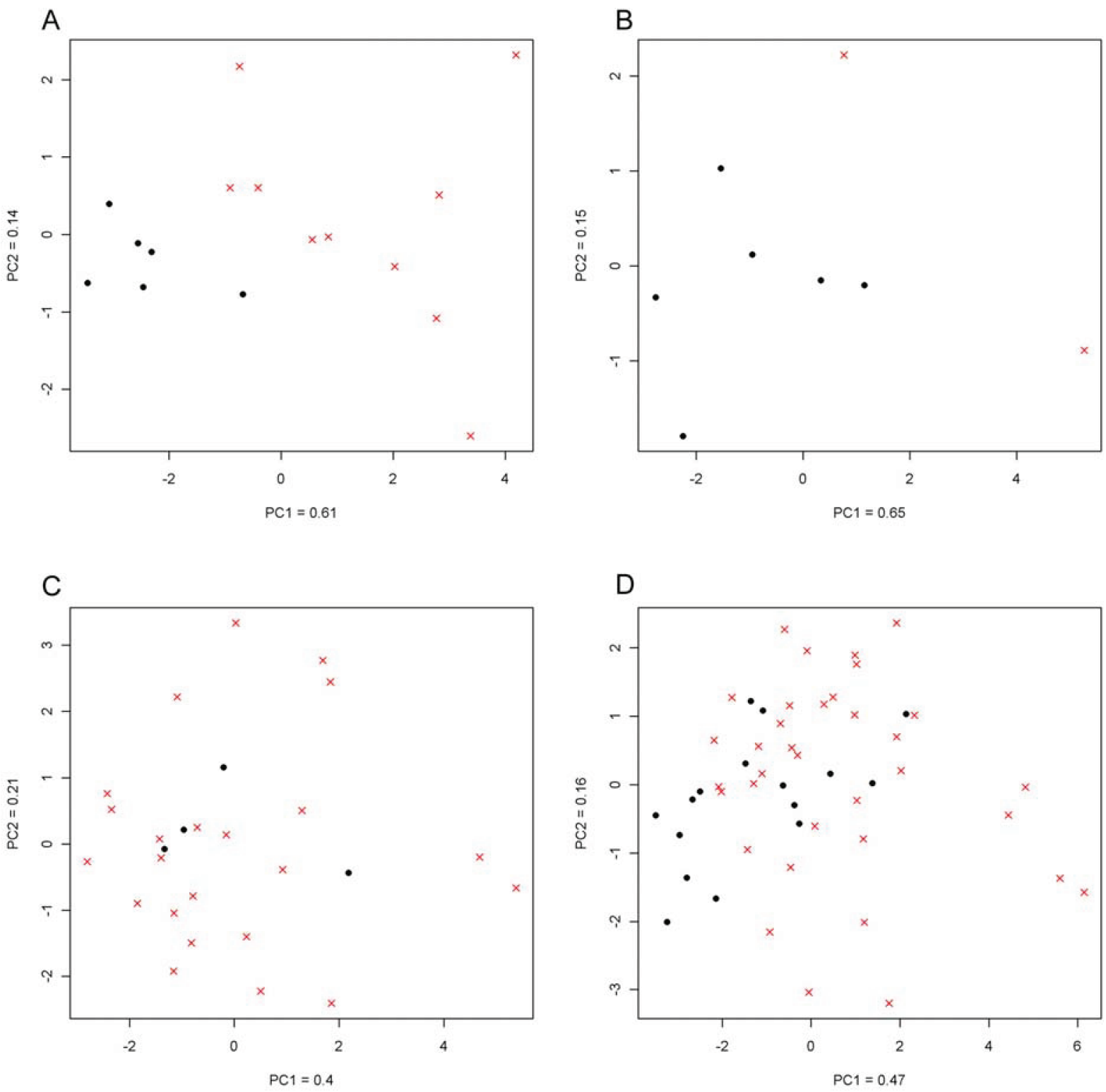

Figure 3. PCA of 10 significant metabolites. (A) Group A, nonsmokers and nondrinkers; (B) group B, drinkers but not smokers; (C) group C, smokers; and (D) all study participants. - , controls; and $\times$, welders.

We further examined the association of these 10 metabolites with welding fume exposure among smokers and drinkers. In individuals of group B, who were alcohol users but not smokers, the trend in concentrations of these 10 metabolites between the welders and the controls (Figure $\mathrm{S} 1$ in the Supporting Information) was similar to that of group A (participants refraining from alcohol and cigarette use), except for acetone and creatinine. This may explain why PCA shows a distinct pattern of the 10 metabolites between the welders and the controls in group B (Figure 3B). Smoking, on the other hand, appeared to have a larger impact on metabolite concentration, where PCA of the 10 metabolites revealed a substantial overlap in individuals of group $\mathrm{C}$ between the welders and the controls, suggesting similar concentrations of the 10 metabolites among smokers (Figure 3C and Figure S2 in the Supporting Information). Therefore, it is not surprising that the PCA from all 51 participants showed a mix-up pattern between the welders and the controls (Figure 3D).

\section{DISCUSSION}

Although the compositions of welding fumes are dependent on welding materials, processes, and the types of shield gases, our study detected environmental concentrations of chromium, nickel, and manganese from two different types of samplers (Table 2). Chromium and nickel are considered to be carcinogenic metals and can elicit inflammatory immune responses in welders. ${ }^{44}$ Also, high brain manganese levels and the inhalation of manganese-containing welding fumes have been observed to induce oxidative stress and inflammation in rat. ${ }^{45,46}$ In general, welding fumes can be viewed as oxidative pollutants and can produce adverse health effects on welders through increased oxidative stress. ${ }^{47,48}$ The challenges to understand the adverse effects of welding fume exposure on human health are the complex nature of environmental influences on human health and the diverse medical history of each welder. Major health behaviors that could greatly affect the study participants' metabolic profiles included smoking, alcohol use, and prior medical history. Therefore, we began our 
metabolomic analysis by analyzing data from study participants who were nonsmokers and nonalcohol users. We then investigated the effect of smoking and alcohol use in the consequent analysis.

Using metabolomics approach, 10 metabolites were identified (Table 3 ) that had significantly different concentrations between the welders and the controls after excluding participants with smoking and drinking habits. Most of the metabolites, including glycine, gluconate, hippurate, $S$-sulfocystein, taurine, TMAO/betaine, and creatinine, were increased significantly in the welders. The only metabolite that was significantly decreased in the welders was creatine. These metabolites are involved in carbohydrate metabolism, amino acid metabolism, oxidation/reduction pathways, and urea metabolism.

We found significant increases of taurine, glycine, and betaine in the welders. With existing evidence of the correlation between welding fume exposure and oxidative stress, these metabolites may be important in modulating inflammatory processes and oxidative tissue injury. Taurine possesses antioxidative properties that can efficiently scavenge ROS at a physiological concentration. ${ }^{49}$ The significant increase of taurine concentration and mild inflammation found from cytokine assays in welders suggests that high oxidative stress in welders causes increased ROS production, thus promoting taurine release. Glycine modulates immune function and various types of tissue injury ${ }^{50}$ by stabilizing the plasma membrane, suppressing increased intracellular calcium, and preventing necrotic cell death-resulting in cytoprotective effects. ${ }^{51}$ In human neutrophils, glycine was able to inhibit ROS production. ${ }^{52}$ A similar antioxidative effect is also found with betaine. Betaine restores antioxidant enzyme (paraoxonase) and glutathione levels in addition to quenching free radicals to alleviate liver injury. ${ }^{53}$ Our study indicates that increased levels of glycine and betaine in welders may be a self-protective mechanism against environmental stress.

In contrast to the other identified metabolites, creatine concentrations were significantly decreased among the welders in our study. ROS and free radicals decrease creatine kinase activity both directly and indirectly. ${ }^{54,55}$ Because welding fumes produce oxidative stress, we suspect that ROS decreased creatine kinase and therefore caused a decrease in creatine concentration. The proposed relationship of ROS and metabolites affected by welding fume exposure is summarized in Figure 4. However, further studies are necessary as subsequent work to verify the biochemical roles of the proposed mechanism in response to welding exposure.

The mechanisms of increasing levels of serine, acetone, gluconate, TMAO, creatinine, and $S$-sulfocysteine among welders remained unclear. Although serine is the most significant metabolite identified in this study, it plays complex biochemical roles in human body such as maintaining health, preventing disease, and cell proliferation. ${ }^{56}$ The mechanism of high urinary serine concentrations among welders needs to be further investigated. We also found significantly increased hippurate levels in the welders. Urine hippurate is a common biological marker commonly utilized to monitor toluene exposure in the working environment or is a marker related gut microflora. The literature suggests that urine hippurate levels correlate with the degree of toluene exposure in spraypainting environments, ${ }^{57}$ hinting that increased hippurate concentrations in our welders might be a result of their exposure to toluene solvents. However, no overuse of toluene

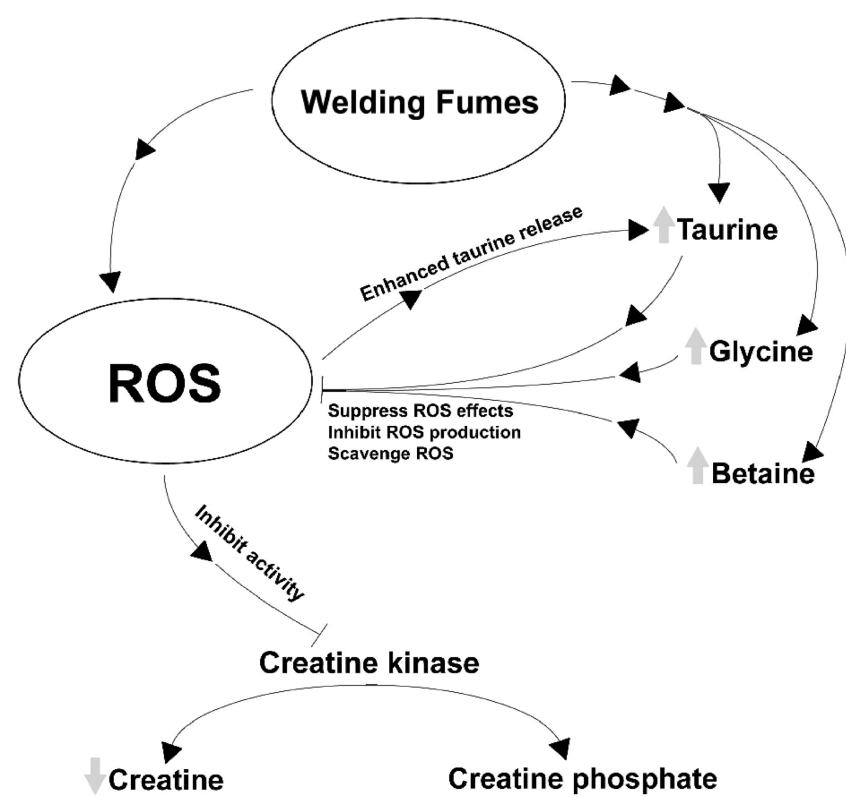

Figure 4. Relationship of ROS and metabolites affected by welding fume exposure.

in this site has been reported. The increased urine hippurate level in the welders remains further investigation.

It is worth mentioning that some of the metabolites identified, such as creatine, hippurate, taurine, creatinine, and TMAO, are suggested to be the usual biomarkers of other toxins discovered in previous metabolomic studies. ${ }^{58}$ Therefore, their alterations have been criticized for being nonspecific in metabolomic investigations. Other than all metabolites being observed for welding fume exposures, we speculate more specific metabolites for welding fumes exposure to be discovered in future studies or by applying more sensitive mass spectrometry-based metabolomics.

We also examined the association of several inflammatory markers with welding exposure. Animals treated with soluble chromium captured from welding fumes showed inflammation alterations and increased cytokines (TNF- $\alpha$, IL-6) that are known to be involved in lung immune responses. ${ }^{59}$ However, of the cytokines and other inflammatory markers examined in our study participants, only TNF- $\alpha$ displayed distinct expression levels between the exposure and the nonexposure groups. The pro-inflammatory cytokine TNF- $\alpha$, which participates in initiation and maintenance of inflammation, controls inflammatory cells as well as mediates many inflammatory processes. Therefore, the up-regulation of TNF was linked to an immune response.

However, the reason why only TNF- $\alpha$ was increased but no other inflammatory markers might due to the fact that with the long history of exposure, the welders may adapt or resist to the welding fumes exposure. The previous study also mentioned that IL-6 was regulated in part by other anti-inflammatory cytokine to reduce the expression and function of the proinflammatory cytokine signals, ${ }^{60}$ indicating that cells have the potential to autoregulate the inflammatory status and decrease the expression level of inflammatory markers.

In summary, we observed that (1) only TNF- $\alpha$ increased significantly, (2) the amount of leukocytes in nasal lavage did not increase, and (3) both fixed point and personal particle sampling of welding fume concentrations did not exceed the allowable welding fume concentration limit. These observations 
indicated that despite the fact that the employment tenure of most welders in this study exceeded 20 years in the same shipyard, the inflammatory responses were not severe among the sampled welders. Even though the inflammatory responses were not severe among welders, their NMR metabolomic profiles revealed a distinct pattern as compared with the control group. This result indicated that metabolomics is sensitive in detecting subtle changes in response to environmental challenges.

We further determined the concentrations of the 10 welding exposure-associated metabolites were different between the welders and the controls in tobacco smokers and alcohol users (Figure 3). Alcohol causes a wide range of disturbances in cellular/organelle physiology via gene expression alterations that impact the downstream cascade of metabolites. However, the influence of alcohol may depend on dose and duration of alcohol consumption. ${ }^{61}$ Our results demonstrated that alcohol consumption did not substantially alter the association of metabolite concentrations with welding exposure for most of the 10 metabolites (Figure 3B). Caution, however, must be taken when interpreting this result because the number of alcohol users in group B is relatively small. Smoking, on the other hand, affected the concentrations of these 10 metabolites. For smokers, no distinct metabolomic pattern was observed between the welders and the controls (Figure 3C). Interestingly, this masking effect attributable to smoking was similarly observed in a study undertaken at an apprentice welding school. ${ }^{62}$ The white blood count, neutrophil count, and fibrinogen levels were significantly elevated in nonsmokers, but not smokers, exposed to welding fumes. Other carcinogenetic studies have shown that tobacco smoking is an important confounding variable of welding fume exposure. ${ }^{62-65}$ These results and our findings suggest that metabolomic profiles reveal the overall effect of welding fume exposure and other confounders.

In conclusion, this study characterized a series of urinary metabolomic changes associated with welding fume exposure. Our results largely correspond with the results of prior studies that utilized nonmetabolomic analytical techniques, suggesting that the use of metabolomic profiling is a sensitive method to characterize the overall effect of welding fume exposure and other confounders. The strength of metabolomics is the ability to detect metabolic changes prior to more overt signs of toxicity. Nevertheless, further investigation at different welding construction sites with larger populations will provide more insight into utilizing a panel of urinary metabolites to assess welding fume exposure in humans.

\section{ASSOCIATED CONTENT}

\section{S Supporting Information}

Figures of distribution of the 10 metabolites in groups B and C. This material is available free of charge via the Internet at http://pubs.acs.org.

\section{AUTHOR INFORMATION}

\section{Corresponding Author}

*Tel: +886.2.3366.4888 \#529. Fax: +886.2.23628167. E-mail: yjtseng@csie.ntu.edu.tw.

\section{Author Contributions}

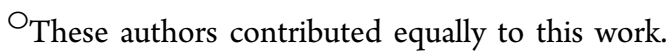

\section{Funding}

This work was funded by the Taiwan National Science Council, Grant 96-2218-E-002-019- and NSC 100-2325-B-002-004-.

\section{Notes}

The authors declare no competing financial interest.

\section{ACKNOWLEDGMENTS}

Resources of the Laboratory of Computational Molecular Design and Detection, Department of Computer Science and Information Engineering, and Graduate Institute of Biomedical Engineering and Bioinformatics of National Taiwan University were used in performing these studies.

\section{REFERENCES}

(1) Nicholson, J. K., Lindon, J. C., and Holmes, E. (1999) 'Metabonomics': Understanding the metabolic responses of living systems to pathophysiological stimuli via multivariate statistical analysis of biological NMR spectroscopic data. Xenobiotica 29, $1181-1189$

(2) Dorman, D. C., Struve, M. F., Norris, A., and Higgins, A. J. (2008) Metabolomic analyses of body fluids after subchronic manganese inhalation in rhesus monkeys. Toxicol. Sci. 106, 46-54.

(3) Fowler, B. A., Conner, E. A., and Yamauchi, H. (2005) Metabolomic and proteomic biomarkers for III-V semiconductors: Chemical-specific porphyrinurias and proteinurias. Toxicol. Appl. Pharmacol. 206, 121-130.

(4) Skordi, E., Yap, I. K., Claus, S. P., Martin, F. P., Cloarec, O., Lindberg, J., Schuppe-Koistinen, I., Holmes, E., and Nicholson, J. K. (2007) Analysis of time-related metabolic fluctuations induced by ethionine in the rat. J. Proteome Res. 6, 4572-4581.

(5) Tsang, T. M., Haselden, J. N., and Holmes, E. (2009) Metabonomic characterization of the 3-nitropropionic acid rat model of Huntington's disease. Neurochem. Res. 34, 1261-1271.

(6) Griffin, J. L., and Shore, R. F. (2007) Applications of metabonomics within environmental toxicology. In The Handbook of Metabonomics and Metabolomics (Lindon, J. C., Nicholson, J. K., Holmes, E., Eds.) pp 517-532, Elsevier, Oxford.

(7) Viant, M. R. (2008) Recent developments in environmental metabolomics. Mol. Biosyst. 4, 980-986.

(8) Hessel, P. A., Melenka, L. S., Michaelchuk, D., Herbert, F. A., and Cowie, R. L. (1998) Lung health among boilermakers in Edmonton, Alberta. Am. J. Ind. Med. 34, 381-386.

(9) Silverstein, M., Maizlish, N., Park, R., and Mirer, F. (1985) Mortality among workers exposed to coal tar pitch volatiles and welding emissions: an exercise in epidemiologic triage. Am. J. Public Health $75,1283-1287$.

(10) Wilson, J. D., Stenzel, M. R., Lombardozzi, K. L., and Nichols, C. L. (1981) Monitoring personnel exposure to stainless steel welding fumes in confined spaces at a petrochemical plant. Am. Ind. Hyg. Assoc. J. 42, 431-436.

(11) Bradshaw, L. M., Fishwick, D., Slater, T., and Pearce, N. (1998) Chronic bronchitis, work related respiratory symptoms, and pulmonary function in welders in New Zealand. Occup. Environ. Med. 55, 150-154.

(12) Brigham, C. R., and Landrigan, P. J. (1985) Safety and health in boatbuilding and repair. Am. J. Ind. Med. 8, 169-182.

(13) Coggon, D., Inskip, H., Winter, P., and Pannett, B. (1994) Lobar pneumonia: An occupational disease in welders. Lancet 344, $41-43$.

(14) Hjortsberg, U., Orbaek, P., and Arborelius, M. Jr. (1992) Small airways dysfunction among non-smoking shipyard arc welders. $\mathrm{Br}$. J. Ind. Med. 49, 441-444.

(15) Lyngenbo, O., Groth, S., Groth, M., Olsen, O., and Rossing, N. (1989) Occupational lung function impairment in never-smoking Danish welders. Scand. J. Soc. Med. 17, 157-164.

(16) McDonald, J. C., Chen, Y., Zekveld, C., and Cherry, N. M. (2005) Incidence by occupation and industry of acute work related 
respiratory diseases in the UK, 1992-2001. Occup. Environ. Med. 62, 836-842.

(17) Gustavsson, P., Jakobsson, R., Johansson, H., Lewin, F., Norell, S., and Rutkvist, L. E. (1998) Occupational exposures and squamous cell carcinoma of the oral cavity, pharynx, larynx, and oesophagus: A case-control study in Sweden. Occup. Environ. Med. 55, 393-400.

(18) Juutilainen, J., Laara, E., and Pukkala, E. (1990) Incidence of leukaemia and brain tumours in Finnish workers exposed to ELF magnetic fields. Int. Arch. Occup. Environ. Health 62, 289-293.

(19) Siew, S. S., Kauppinen, T., Kyyronen, P., Heikkila, P., and Pukkala, E. (2008) Exposure to iron and welding fumes and the risk of lung cancer. Scand. J. Work Environ. Health 34, 444-450.

(20) Sorensen, A. R., Thulstrup, A. M., Hansen, J., Ramlau-Hansen, C. H., Meersohn, A., Skytthe, A., and Bonde, J. P. (2007) Risk of lung cancer according to mild steel and stainless steel welding. Scand. J. Work Environ. Health 33, 379-386.

(21) Afeseh Ngwa, H., Kanthasamy, A., Anantharam, V., Song, C., Witte, T., Houk, R., and Kanthasamy, A. G. (2009) Vanadium induces dopaminergic neurotoxicity via protein kinase Cdelta dependent oxidative signaling mechanisms: relevance to etiopathogenesis of Parkinson's disease. Toxicol. Appl. Pharmacol. 240, 273-285.

(22) Stettler, L. E., Groth, D. H., and MacKay, G. R. (1977) Identification of stainless steel welding fume particulates in human lung and environmental samples using electron probe microanalysis. Am. Ind. Hyg. Assoc. J. 38, 76-82.

(23) van der Wal, J. F. (1990) Exposure of welders to fumes and gases in Dutch industries: summary of results. Ann. Occup. Hyg. 34, 45-54.

(24) Roth, J. A. (2006) Homeostatic and toxic mechanisms regulating manganese uptake, retention, and elimination. Biol. Res. $39,45-57$.

(25) Antonini, J. M. (2003) Health effects of welding. Crit. Rev. Toxicol. 33, 61-103.

(26) Hedenstedt, A., Jenssen, D., Lidestein, B. M., Ramel, C., Rannug, U., and Stern, R. M. (1977) Mutagenicity of fume particles from stainless steel welding. Scand. J. Work Environ. Health 3, 203211.

(27) Keskinen, H., Kalliomaki, P. L., and Alanko, K. (1980) Occupational asthma due to stainless steel welding fumes. Clin. Allergy 10, 151-159.

(28) Sriram, K., Lin, G. X., Jefferson, A. M., Roberts, J. R., Chapman, R. S., Chen, B. T., Soukup, J. M., Ghio, A. J., and Antonini, J. M. (2010) Dopaminergic neurotoxicity following pulmonary exposure to manganese-containing welding fumes. Arch. Toxicol. 84, 521-540.

(29) Tessier, D. M., and Pascal, L. E. (2006) Activation of MAP kinases by hexavalent chromium, manganese and nickel in human lung epithelial cells. Toxicol. Lett. 167, 114-121.

(30) Rim, K. T., Park, K. K., Sung, J. H., Chung, Y. H., Han, J. H., Cho, K. S., Kim, K. J., and Yu, I. J. (2004) Gene-expression profiling using suppression-subtractive hybridization and cDNA microarray in rat mononuclear cells in response to welding-fume exposure. Toxicol. Ind. Health 20, 77-88.

(31) Chuang, C. H., Huang, C. E., and Chen, H. L. (2010) DNA strand breakage and lipid peroxidation after exposure to welding fumes in vivo. Mutagenesis 25, 71-76.

(32) Iarmarcovai, G., Sari-Minodier, I., Orsiere, T., De Meo, M., Gallice, P., Bideau, C., Iniesta, D., Pompili, J., Berge-Lefranc, J. L., and Botta, A. (2006) A combined analysis of XRCC1, XRCC3, GSTM1 and GSTT1 polymorphisms and centromere content of micronuclei in welders. Mutagenesis 21, 159-165.

(33) Iarmarcovai, G., Sari-Minodier, I., Chaspoul, F., Botta, C., De Meo, M., Orsiere, T., Berge-Lefranc, J. L., Gallice, P., and Botta, A. (2005) Risk assessment of welders using analysis of eight metals by ICP-MS in blood and urine and DNA damage evaluation by the comet and micronucleus assays; influence of XRCC1 and XRCC3 polymorphisms. Mutagenesis 20, 425-432.

(34) Liu, H. H., Wu, Y. C., and Chen, H. L. (2007) Production of ozone and reactive oxygen species after welding. Arch. Environ. Contam. Toxicol. 53, 513-518.
(35) Cheng, T. J., Kao, H. P., Chan, C. C., and Chang, W. P. (2003) Effects of ozone on DNA single-strand breaks and 8-oxoguanine formation in A549 cells. Environ. Res. 93, 279-284.

(36) McNeilly, J. D., Heal, M. R., Beverland, I. J., Howe, A., Gibson, M. D., Hibbs, L. R., MacNee, W., and Donaldson, K. (2004) Soluble transition metals cause the pro-inflammatory effects of welding fumes in vitro. Toxicol. Appl. Pharmacol. 196, 95-107.

(37) Prabhakaran, K., Ghosh, D., Chapman, G. D., and Gunasekar, P. G. (2008) Molecular mechanism of manganese exposure-induced dopaminergic toxicity. Brain Res. Bull. 76, 361-367.

(38) Gube, M., Ebel, J., Brand, P., Goen, T., Holzinger, K., Reisgen, U., and Kraus, T. (2010) Biological effect markers in exhaled breath condensate and biomonitoring in welders: Impact of smoking and protection equipment. Int. Arch. Occup. Environ. Health 83, 803-811.

(39) Hsiech, C. M. (2006) Heavy metal exposure assessment of steel welding workers, p 13, Institute of Occupational Safety and Health Council of Labor Affairs, Taiwan.

(40) Naclerio, R. M., Meier, H. L., Kagey-Sobotka, A., Adkinson, N. F. Jr., Meyers, D. A., Norman, P. S., and Lichtenstein, L. M. (1983) Mediator release after nasal airway challenge with allergen. Am. Rev. Respir. Dis. 128, 597-602.

(41) Prat, J., Xaubet, A., Mullol, J., Plaza, V., Maso, M., Lleonart, R., and Picado, C. (1993) Immunocytologic analysis of nasal cells obtained by nasal lavage: A comparative study with a standard method of cell identification. Allergy 48, 587-591.

(42) JD, S. (2003) The positive false discovery rate: A Bayesian interpretation and the q-value. Ann. Stat. 31, 2013-2035.

(43) Gartland, K. P., Beddell, C. R., Lindon, J. C., and Nicholson, J. K. (1991) Application of pattern recognition methods to the analysis and classification of toxicological data derived from proton nuclear magnetic resonance spectroscopy of urine. Mol. Pharmacol. 39, 629642 .

(44) Frenkel, K., Karkoszka, J., Cohen, B., Baranski, B., Jakubowski, M., Cosma, G., Taioli, E., and Toniolo, P. (1994) Occupational exposures to $\mathrm{Cd}, \mathrm{Ni}$, and $\mathrm{Cr}$ modulate titers of antioxidized DNA base autoantibodies. Environ. Health Perspect. 102 (Suppl. 3), 221-225.

(45) Erikson, K. M., Dobson, A. W., Dorman, D. C., and Aschner, M. (2004) Manganese exposure and induced oxidative stress in the rat brain. Sci. Total Environ. 334-335, 409-416.

(46) Sriram, K., Lin, G. X., Jefferson, A. M., Roberts, J. R., Chapman, R. S., Chen, B. T., Soukup, J. M., Ghio, A. J., and Antonini, J. M. (2010) Dopaminergic neurotoxicity following pulmonary exposure to manganese-containing welding fumes. Arch. Toxicol. 84, 521-540.

(47) Han, S. G., Kim, Y., Kashon, M. L., Pack, D. L., Castranova, V., and Vallyathan, V. (2005) Correlates of oxidative stress and freeradical activity in serum from asymptomatic shipyard welders. Am. J. Respir. Crit. Care Med. 172, 1541-1548.

(48) Bagchi, D., Stohs, S. J., Downs, B. W., Bagchi, M., and Preuss, H. G. (2002) Cytotoxicity and oxidative mechanisms of different forms of chromium. Toxicology 180, 5-22.

(49) Oliveira, M. W., Minotto, J. B., de Oliveira, M. R., Zanotto-Filho, A., Behr, G. A., Rocha, R. F., Moreira, J. C., and Klamt, F. (2010) Scavenging and antioxidant potential of physiological taurine concentrations against different reactive oxygen/nitrogen species. Pharmacol. Rep. 62, 185-193.

(50) Zhong, Z., Wheeler, M. D., Li, X., Froh, M., Schemmer, P., Yin, M., Bunzendaul, H., Bradford, B., and Lemasters, J. J. (2003) LGlycine: A novel antiinflammatory, immunomodulatory, and cytoprotective agent. Curr. Opin. Clin. Nutr. Metab. Care 6, 229-240.

(51) Ascher, E., Hanson, J. N., Cheng, W., Hingorani, A., and Scheinman, M. (2001) Glycine preserves function and decreases necrosis in skeletal muscle undergoing ischemia and reperfusion injury. Surgery 129, 231-235.

(52) Giambelluca, M. S., and Gende, O. A. (2009) Effect of glycine on the release of reactive oxygen species in human neutrophils. Int. Immunopharmacol. 9, 32-37.

(53) Varatharajalu, R., Garige, M., Leckey, L. C., Gong, M., and Lakshman, M. R. (2010) Betaine protects chronic alcohol and omega3 PUFA-mediated down-regulations of PON1 gene, serum PON1 and 
homocysteine thiolactonase activities with restoration of liver GSH. Alcohol.: Clin. Exp. Res. 34, 424-431.

(54) Genet, S., Kale, R. K., and Baquer, N. Z. (2000) Effects of free radicals on cytosolic creatine kinase activities and protection by antioxidant enzymes and sulfhydryl compounds. Mol. Cell. Biochem. 210, 23-28.

(55) Koufen, P., Ruck, A., Brdiczka, D., Wendt, S., Wallimann, T., and Stark, G. (1999) Free radical-induced inactivation of creatine kinase: Influence on the octameric and dimeric states of the mitochondrial enzyme (Mib-CK). Biochem. J. 344 (Part 2), 413-417.

(56) de Koning, T. J., Snell, K., Duran, M., Berger, R., Poll-The, B. T., and Surtees, R. (2003) L-serine in disease and development. Biochem. J. 371, 653-661.

(57) Amorim, L. C., and Alvarez-Leite, E. M. (1997) Determination of o-cresol by gas chromatography and comparison with hippuric acid levels in urine samples of individuals exposed to toluene. J. Toxicol. Environ. Health 50, 401-407.

(58) Robertson, D. G. (2005) Metabonomics in toxicology: A review. Toxicol. Sci. 85, 809-822.

(59) Antonini, J. M., and Roberts, J. R. (2007) Chromium in stainless steel welding fume suppresses lung defense responses against bacterial infection in rats. J. Immunotoxicol. 4, 117-127.

(60) de Waal Malefyt, R., Abrams, J., Bennett, B., Figdor, C. G., and de Vries, J. E. (1991) Interleukin 10(IL-10) inhibits cytokine synthesis by human monocytes: An autoregulatory role of IL-10 produced by monocytes. J. Exp. Med. 174, 1209-1220.

(61) Uddin, R. K., Treadwell, J. A., and Singh, S. M. (2005) Towards unraveling ethanol-specific neuro-metabolomics based on ethanol responsive genes in vivo. Neurochem. Res. 30, 1179-1190.

(62) Danielsen, T. E., Langard, S., and Andersen, A. (1998) Incidence of lung cancer among shipyard welders investigated for siderosis. Int. J. Occup. Environ. Health 4, 85-88.

(63) Danielsen, T. E., Langard, S., Andersen, A., and Knudsen, O. (1993) Incidence of cancer among welders of mild steel and other shipyard workers. Br. J. Ind. Med. 50, 1097-1103.

(64) Danielsen, T. E., Langard, S., and Andersen, A. (2000) Incidence of cancer among welders and other shipyard workers with information on previous work history. J. Occup. Environ. Med. 42, 101109.

(65) Danielsen, T. E., Langard, S., and Andersen, A. (1996) Incidence of cancer among Norwegian boiler welders. Occup. Environ. Med. 53, 231-234. 